manuscript No.

(will be inserted by the editor)

\title{
On the response of autonomous sweeping processes to periodic perturbations
}

\author{
Mikhail Kamenskii • Oleg Makarenkov
}

Received: date / Accepted: date

\begin{abstract}
If $x_{0}$ is an equilibrium of an autonomous differential equation $\dot{x}=$ $f(x)$ and $\operatorname{det}\left\|f^{\prime}\left(x_{0}\right)\right\| \neq 0$, then $x_{0}$ persists under autonomous perturbations and $x_{0}$ transforms into a $T$-periodic solution under non-autonomous $T$-periodic perturbations. In this paper we discover a similar structural stability for Moreau sweeping processes of the form $-\dot{u} \in N_{B}(u)+f_{0}(u), u \in \mathbb{R}^{2}$, i. e. we consider the simplest case where the derivative is taken with respect to the Lebesgue measure and where the convex set $B$ of the reduced system is a non-moving unit ball of $\mathbb{R}^{2}$. We show that an equilibrium $\left\|u_{0}\right\|=1$ persists under periodic perturbations, if the projection $\bar{f}: \partial B \rightarrow \mathbb{R}^{2}$ of $f_{0}$ on the tangent to the boundary $\partial B$ is nonsingular at $u_{0}$.
\end{abstract}

Keywords Sweeping process · perturbation theory · continuation principle · periodic solution

Mathematics Subject Classification (2000) 34A60 $\cdot 70 \mathrm{H} 12 \cdot 37 \mathrm{C} 25$

\section{Introduction}

We investigate the dynamics of a particle $u(t) \in \mathbb{R}^{2}$ which is governed by the differential equation

$$
-\dot{u}(t)=f(t, u(t)), \quad \text { if }\|u(t)\|<1,
$$

M. Kamenskii

BCAM - Basque Center for Applied Mathematics, Mazarredo 14, E-48009, Bilbao, Basque Country, Spain

E-mail: mikhailkamenski@mail.ru

Present address: Department of Mathematics, Voronezh State University, 394006 Voronezh, Russia

O. Makarenkov

Department of Mathematical Sciences, University of Texas at Dallas, 75080 Richardson, USA

E-mail: makarenkov@utdallas.edu 
and which satisfies the differential inclusion

$$
-\dot{u}(t) \in \bigcup_{\lambda \in[0, \infty)} \lambda u(t)+f(t, u(t)), \quad \text { if }\|u(t)\| \geq 1 .
$$

Fig. 1 illustrates the two situations.
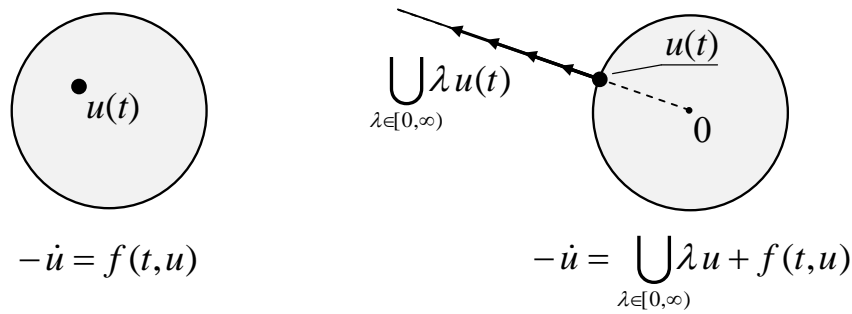

Fig. 1 The occurrence of an unbounded vector field when $u(t)$ reaches the boundary $\partial B$ of $B$ (colored in gray).

System (1)-(2) is the simplest Moreau sweeping process, which is usually formulated as

$$
-\dot{u}(t) \in N_{B}(u(t))+f(t, u(t))
$$

where

$$
N_{C}(x)=\left\{\xi \in \mathbb{R}^{2}:\langle\xi, c-x\rangle \leq 0, \text { for any } c \in C\right\},
$$

is the so-called outward normal cone to a closed convex set $C \in \mathbb{R}^{2}$ and $B=\left\{x \in \mathbb{R}^{2}:\|x\| \leq 1\right\}$. The solution of (3) is understood in the classical sense (see e.g. $[1,13]$ ), i.e. an absolutely continuous function $u:[0, T] \mapsto \mathbb{R}^{2}$ is said to be a solution of (3), if $u$ satisfies (3) for almost all $t \in[0, T]$. The existence of such a solution doesn't follow from the theory of differential inclusions because the right-hand-side in (3) is unbounded. The respective theory for sweeping processes has its roots in the pioneering work of Moreau [20], who proposed a discrete approximation of (3) that converges to a solution $u$, see also Monteiro-Marques [19, Theorem 2.1] and Castaing \& Monteiro-Marques [10]. In addition, the work [20] states that the solution $u$ is unique in the class of right-continuous functions with values in $B$.

Sweeping process (3) with Lipschitz $f$ and convex Lipschitz $t \mapsto B(t)$ is a convenient framework to construct solutions of equations of elastoplasticity (see Moreau [21], Kunze \& Monteiro-Marques [17] and references therein). The case of a polyhedral set $B$ provides a powerful deterministic tool towards the Skorokhod problem for a reflecting Brownian motion (Krejci \& Vladimirov [15]). Multi-functions $f$ and $t \mapsto B(t)$ of bounded variation BV (i.e. a way less regular that the Lipschitz ones) and respective Radon-Nikodym derivatives appear in the context of mechanical problems with frictional contacts where the differential equations framework turns out to be ill-posed. The best example is the resolution of the Painlevé paradox by Stewart [27], where a Moreau 
discretization scheme is used too. Sweeping process (3) with a BV moving set $t \mapsto B(t)$ describes repeated collisions in vibro-impact problems, see Paoli [22]. The reader is referred to the book by Brogliato [6] for introduction and further references. A possible way to investigate the solutions of the BV sweeping processes is through a suitable Lipschitz approximation (Valadier [32], see also Monteiro-Marques [19, §5.2]). Recupero [25] transforms a BV sweeping process into a Lipschitz one by means of a suitable reparameterization.

We stick to a $C^{1}$-smooth $f$ and a non-moving ball $B$ in order to avoid technical details and to make the essential contribution of our work clear. Our proofs carry over in the case where $f$ is Lipschitz and $t \mapsto B(t)$ is an arbitrary convex set Lipschitz in $t$.

The theory of Moreau sweeping processes has been rapidly developing over the recent years. Since the celebrated results by Moreau [20] about the convergence of a discrete scheme to a solution of $(3)$ (see also $[2,17]$, where the set $B$ is state dependent), there were several important contributions towards the dependence of solutions of (3) on initial conditions (Castaing \& MonteiroMarques [10], Paoli [24], Ballard [3], Krejci \& Roche [16]) and the moving set (Kunze \& Monteiro-Marques [17]). There were further developments on relaxing the regularity of time- and state- dependent function $B$, on dropping the convexity assumption on $B$, on replacing the cone $K_{B}(u)$ by more general convex sets, etc., see Thibault [30], Bounkhel \& Thibault [5], Tolstonogov [31], Stewart [29]. A significant attention in the literature has been devoted to identifying relevant structures in Moreau sweeping processes, primarily periodic solutions, see e.g. the above-mentioned papers [17] and [10]. A Lyapunov functions approach capable to study Lyapunov stability of periodic solutions of (3) has been developed as well (see Brogliato [6], Leine \& Wouw [18] and references therein).

The goal of this paper is to make a significant step towards understanding the structural stability of Moreau sweeping processes. Specifically, we identify a prototypic Moreau sweeping process (3) that possesses a simplest possible periodic solution (i.e. equilibrium) and investigate the response of this periodic solution to periodic perturbations. The result that we propose for the Moreau sweeping processes (3) is similar to the classical results of Berstein \& Halanai [4] and Cronin [11] in the theory of ordinary differential equations. Results $[4,11]$ found many applications and stimulated several studies in geometric perturbation theory (continuation) for ODEs, see Capietto, Mawhin \& Zanolin [9] for details. We, therefore, anticipate that this paper will serve as a starting point for the development of perturbation theory for sweeping processes.

\section{Main result}

Consider an autonomous sweeping process

$$
-\dot{u}(t) \in N_{B}(u(t))+f_{0}(u(t)), \quad u \in \mathbb{R}^{2},
$$


that possesses a boundary equilibrium

$$
u_{*} \in \partial B
$$

i. e. $u_{*}$ verifies

$$
f_{0}\left(u_{*}\right) \in-N_{B}\left(u_{*}\right) \quad \text { and } \quad f_{0}\left(u_{*}\right) \neq 0 .
$$

If $f_{0}\left(u_{*}\right) \neq 0$, then such a boundary equilibrium generically persists under small autonomous perturbations ${ }^{1}$. Our goal is to study the response of $u_{*}$ to $T$-periodic perturbations. Specifically, we consider

$$
-\dot{u}(t) \in N_{B}(u(t))+f_{\varepsilon}(t, u(t)),
$$

where

$$
f_{\varepsilon}(t+T, u) \equiv f_{\varepsilon}(t, u)
$$

and

$$
f_{\varepsilon}(t, u) \rightarrow f_{0}(u) \quad \text { as } \quad \varepsilon \rightarrow 0
$$

uniformly in $t \in[0, T]$ and $u \in B$. To achieve the goal, we are going to prove the existence of an invariant region for the Poincaré map $P_{\varepsilon}$ of (7) near $u_{*}$. This can be viewed as a version of the classical Berstein-Halanay result [4] or Cronin [11] available for ordinary differential equations.

The Poincaré map $P_{\varepsilon}(\xi)$ is the value at time $t=T$ of the solution of (7) with the initial condition $u(0)=\xi \in B$. The existence and continuity of $P_{\varepsilon}$ follows from Castaing \& Monteiro Marques [10, theorem 4.4].

Let $N: \mathbb{R} \rightarrow \mathbb{R}^{2}$ be some $C^{1}$ parameterization of $\partial B$ in the neighborhood of $u_{*}$, so that $u_{*}=N(0)$. We assume that the parameterization $N$ is positive, i.e. $N(r)$ moves counterclockwise as $r$ increases. For a vector $u \in \mathbb{R}^{2}$, we denote $u^{\perp}=\left(\begin{array}{l}u_{2} \\ -u_{1}\end{array}\right)$. In this section we prove that the $B$ part of the $\delta$-neighborhood of $u_{*}$ (see Fig. 2) is invariant for $P_{\varepsilon}$ for small $\varepsilon>0$ and $\delta>0$. The main role in our analysis is played by the following (bifurcation) function

$$
\bar{f}(r) \mapsto\left\langle f_{0}(N(r)), N(r)^{\perp}\right\rangle .
$$

Property (6) implies that $\bar{f}(0)=0$. Our main result is that nondegeneracy of $\bar{f}$ near 0 is crucial for the persistence of $u_{*}$ under periodic perturbations. The next theorem is a rigorous formulation of this assertion. In what follows, the notation $[x, y]$ stays for the closed line segment of $\mathbb{R}^{2}$ that connects $x$ and $y$.

\footnotetext{
1 The implicit function theorem ensures that $\left\langle f_{\varepsilon}\left(u_{\varepsilon}\right), u_{\varepsilon}^{\perp}\right\rangle=0$ for some $u_{\varepsilon} \rightarrow u_{*}$ as $\varepsilon \rightarrow 0$, if $f_{\varepsilon} \rightarrow f_{0}$ as $\varepsilon \rightarrow 0$ generically (i.e. when the respective determinant at $\varepsilon=0$ doesn't vanish). Here $u^{\perp}=\left(u_{2},-u_{1}\right)^{T}$.
} 


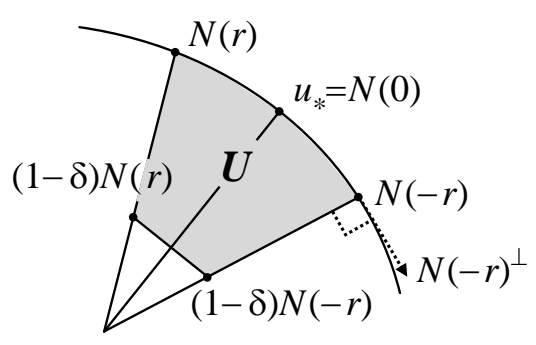

Fig. 2 Black arc: a part of $\partial B$ in the neighborhood of $u_{*}$. Gray set: a $\delta$-neighborhood of $u_{*}$ restricted to the set $B$.

Theorem 1 Let $f_{\varepsilon}$ be $C^{1}$ for all $\varepsilon \in \mathbb{R}$ and assume that $f_{\varepsilon}(t, u) \rightarrow f_{0}(u)$ as $\varepsilon \rightarrow 0$ uniformly in $t \in[0, T]$ and $u \in B$. Consider a positive parameterization $r \mapsto N(r)$ of $\partial B$ such that $u_{*}=N(0)$ is an equilibrium of (4), i.e. (6) holds. If the function $\bar{f}$ is strictly decreasing at 0 , then, for any $r>0$ sufficiently small, there exists $\delta_{0}>0$ such that, given any $\delta \in\left[0, \delta_{0}\right]$, one can find $\varepsilon_{0}>0$ so that

$$
P_{\varepsilon}(U) \subset U, \quad \text { for all } \varepsilon \in\left[0, \varepsilon_{0}\right] \text {, }
$$

where

$$
U=\bigcup_{\lambda \in[0,1]}[\lambda(1-\delta) N(-r)+(1-\lambda)(1-\delta) N(r), N(-\lambda r+(1-\lambda) r)] .
$$

Moreover, none of the solutions of (7) with $u(0) \in U$ leave $U$ through $[0, T]$.

The following lemma is required for the proof of the theorem.

Lemma 1 Let $x_{0} \in \partial B$ and let $N \in \mathbb{R}^{2}$ be the unit normal vector to $\partial B$ at $x_{0}$ pointing outwards $B$. Assume that for $g \in C^{1}\left(\mathbb{R} \times \mathbb{R}^{m}, \mathbb{R}^{2}\right)$ we have

$$
g\left(t_{\mu}, \mu\right)=0 \quad \text { and } \quad\left\langle g_{t}^{\prime}\left(t_{\mu}, \mu\right), N\right\rangle \cdot\left\langle g_{t}^{\prime}\left(t_{\mu}, \mu\right), N^{\perp}\right\rangle \neq 0 \quad \text { for all } \quad \mu \in M,
$$

where $M \subset \mathbb{R}^{m}$ is a compact set and $\mu \mapsto t_{\mu}$ is a continuous function. Then there exists $\Delta t>0$, such that for any $\xi \in\left[0, x_{0}\right]$ and any $\mu \in M$, the solution $v$ of

$$
\left\{\begin{array}{l}
-\dot{v}(t) \in N_{B+g(t, \mu)}(v(t)) \\
v\left(t_{\mu}\right)=\xi
\end{array}\right.
$$

verifies

$$
\left|\left\langle N^{\perp}, v(t)-\xi\right\rangle\right| \leq\|g(t, \mu)\| \cdot\left|\left\langle N^{\perp}, g(t, \mu)\right\rangle\right|, \quad \text { for all } t \in\left[t_{\mu}, t_{\mu}+\Delta t\right] .
$$

Figures 3 and 4 illustrate the notations of this statement as well as all the notations throughout the proof. 


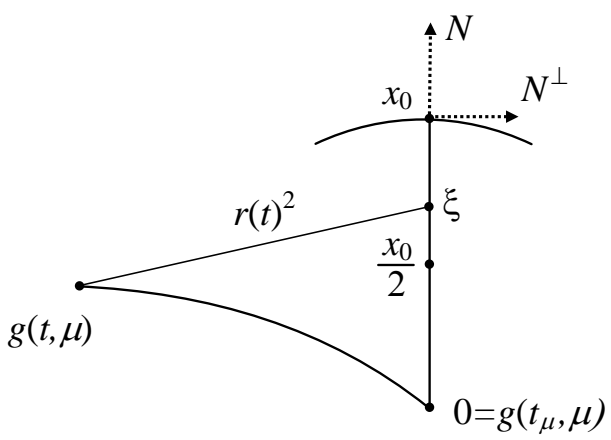

Fig. 3 Illustration of the proof when $\left\langle g^{\prime}\left(t_{\mu}, \mu\right), N\right\rangle>0$.

Proof. The case $\left\langle g_{t}^{\prime}\left(t_{\mu}, \mu\right), N\right\rangle>0$ for all $\mu \in M$. The square of the distance between $\xi \in\left[0, x_{0}\right]$ and $g(t, \mu)$ can be computed as

$$
r(t)=\left\langle g(t, \mu), N^{\perp}\right\rangle^{2}+\langle\xi-g(t, \mu), N\rangle^{2}
$$

(see Fig. 3). One has

$$
r^{\prime}(t)=2\left\langle g(t, \mu), N^{\perp}\right\rangle \cdot\left\langle g_{t}^{\prime}(t, \mu), N^{\perp}\right\rangle+2\langle\xi-g(t, \mu), N\rangle \cdot\left\langle-g_{t}^{\prime}(t, \mu), N\right\rangle
$$

and so

$r^{\prime}\left(t_{\mu}\right)=-2\langle\xi, N\rangle \cdot\left\langle g_{t}^{\prime}\left(t_{\mu}, \mu\right), N\right\rangle \leq-\min _{\mu \in M}\left\langle g_{t}^{\prime}\left(t_{\mu}, \mu\right), N\right\rangle$ for all $\xi \in\left[\frac{x_{0}}{2}, x_{0}\right]$.

This property allows to define $\Delta \mathbf{t}$ as follows: Let us choose $\Delta t>0$ such that $r(t)$ doesn't increase on $\left[t_{\mu}, t_{\mu}+\Delta t\right]$. Therefore,

$$
\|\xi-g(t, \mu)\|^{2} \leq 1, \quad \text { for all } \xi \in\left[\frac{x_{0}}{2}, x_{0}\right], t \in\left[t_{\mu}, t_{\mu}+\Delta t\right], \mu \in M .
$$

By diminishing $\boldsymbol{\Delta} \mathbf{t}>\mathbf{0}$ to ensure that

$$
\|\xi-g(t, \mu)\|^{2} \leq 1, \quad \text { for all } \xi \in\left[0, \frac{x_{0}}{2}\right], t \in\left[t_{\mu}, t_{\mu}+\Delta t\right], \mu \in M,
$$

we get $\Delta t>0$ such that all the points of $\left[0, x_{0}\right]$ lie inside $B+g(t, \mu)$ when $t \in\left[t_{\mu}, t_{\mu}+\Delta t\right]$ and $\mu \in M$, i.e. $v(t)-\xi$ stays identically zero on $\left[t_{\mu}, t_{\mu}+\Delta t\right]$ in this case.

The case $\left\langle g_{t}^{\prime}\left(t_{\mu}, \mu\right), N\right\rangle<0$ for all $\mu \in M$. Defining $\Delta$ t initially: let $\Delta t>0$ be such that

$$
\|g(t, \mu)\| \leq 1 \quad \text { for all } t \in\left[t_{\mu}, t_{\mu}+\Delta t\right], \mu \in M \text {. }
$$

Reduce $\Delta \mathbf{t}>\mathbf{0}$ until

$\langle g(t, \mu), N\rangle$ strictly decreases on $\left[t_{\mu}, t_{\mu}+\Delta t\right]$, for any $\mu \in M$,

$\left\langle g(t, \mu), N^{\perp}\right\rangle$ is strictly monotone on $\left[t_{\mu}, t_{\mu}+\Delta t\right]$, for any $\mu \in M$. 


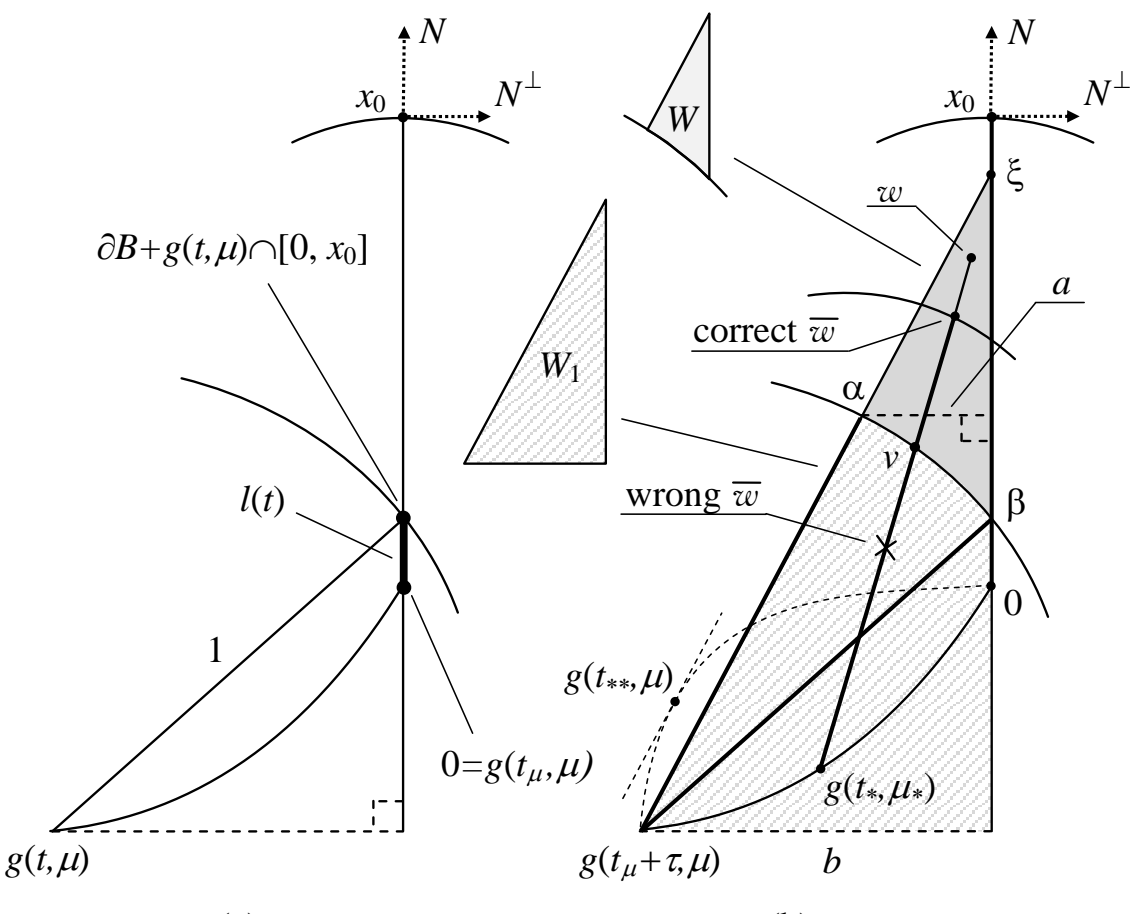

(a)

(b)

Fig. 4 (a) The line segment that measures the distance of the point $\partial B+g(t, \mu) \cap\left[0, x_{0}\right]$ to the origin. (b) The image of point $w$ under the mapping $w \mapsto \operatorname{proj}\left(w, B+g\left(t_{\mu}+\tau, \mu\right)\right)$ (and all the notations we use throughout the proof of lemma 1).

Then the function

$$
l(t)=\sqrt{1-\left\langle N^{\perp}, g(t, \mu)-g\left(t_{\mu}, \mu\right)\right\rangle^{2}}-\left\langle N, g\left(t_{\mu}, \mu\right)-g(t, \mu)\right\rangle
$$

is 1) well defined and strictly positive, and 2) strictly decreasing on $\left[t_{\mu}, t_{\mu}+\Delta t\right]$ starting from $l\left(t_{\mu}\right)=1$. This is equivalent to saying that (see Fig. 4a)

1) unit ball centered at $g(t, \mu)$ intersects $\left[0, x_{0}\right]$ (at the point $l(t) N$ )

2) the above mentioned point of intersection $(\partial B+g(t, \mu)) \cap\left[0, x_{0}\right]$ moves outwards $x_{0}$ strictly monotonically when $t$ increases from $t_{\mu}$ to $t_{\mu}+\Delta t$.

Consider $\xi \in\left[0, x_{0}\right], \tau \in(0, \Delta t]$, and $\mu \in M$.

If $\xi \in B+g\left(t_{\mu}+\tau, \mu\right)$ then $\xi \in B+g(t, \mu)$ for any $t \in\left[t_{\mu}, t_{\mu}+\tau\right]$ by the monotonicity property we just proved, and, therefore, the solution of (8) is the constant $\xi$ on this interval.

Assume that $\xi \notin B+g\left(t_{\mu}+\tau, \mu\right)$. Let $\alpha=\operatorname{proj}\left(\xi, B+g\left(t_{\mu}+\tau, \mu\right)\right)$ and let $\beta=\left(\partial B+g\left(t_{\mu}+\tau, \mu\right)\right) \cap\left[0, x_{0}\right]$, see Fig. 4b. Denote by $W \subset \mathbb{R}^{2}$ the closed set whose boundary is composed by the arc $\alpha \beta$ (denoted by $[\alpha, \beta]$ in the sequel) 
and by the line segments $[\alpha, \xi]$ and $[\xi, \beta]$. We claim that there exists $\Delta t>0$ such that given any $\tau \in(0, \Delta t], \mu \in M$, and $\xi \notin B+g\left(t_{\mu}+\tau, \mu\right)$ the set $W$ is invariant for the map $w \mapsto \operatorname{proj}(w, B+g(t, \mu))$ for all $t \in\left[t_{\mu}, t_{\mu}+\tau\right]$.

Proof: Reducing $\Delta \mathrm{t}$ : let us show that $\Delta t>0$ can be diminished in such a way that

$$
\begin{aligned}
& g_{t}^{\prime}(t, \mu) \nVdash\left[g\left(t_{\mu}+\tau, \mu\right), \xi\right], \\
& \quad \text { for all } t \in\left[t_{\mu}, t_{\mu}+\tau\right], \xi \notin B+g\left(t_{\mu}+\tau, \mu\right), \tau \in(0, \Delta t], \mu \in M .
\end{aligned}
$$

Indeed, assuming that the latter is incorrect, we would get $g_{t}^{\prime}\left(t_{\mu_{\star}}, \mu_{\star}\right) \| N$ for some $\mu_{\star} \in M$, which is impossible per the assumptions of the Lemma.

Property (9) ensures that the line segment $[w, g(t, \mu)]$ belongs to the right triangle formed by the line segment $\left[g\left(t_{\mu}+\tau, \mu\right), \xi\right]$ and its projection on $N$ (denoted by $W_{1}$ at Fig. 4b). If $[w, g(t, \mu)] \not \subset W_{1}$, then the curve $t \mapsto g(t, \mu)$ needs to leave $W_{1}$ at some time moment. This would imply the existence of $t_{* *} \in\left[t_{\mu}, t_{\mu}+\tau\right]$ where $g_{t}^{\prime}\left(t_{* *}, \mu\right)$ is parallel to $\left[g\left(t_{\mu}+\tau, \mu\right), \xi\right]$ (see Fig. $4 \mathrm{~b}$ ), which is excluded by $(9)$.

We are now in the position to prove the statement about the invariance of $W$. Assume the contrary, i.e. assume that there exists $\mu_{*} \in M, w_{*} \in$ $W$ and $t_{*} \in\left[t_{\mu_{*}}, t_{\mu_{*}}+\tau\right]$ such that $\bar{w}=\operatorname{proj}\left(w_{*}, B+g\left(t_{*}, \mu_{*}\right)\right) \notin W$. Since $\bar{w} \in\left[w_{*}, g\left(t_{*}, \mu_{*}\right)\right]$ and, as we noticed earlier, $\left[w_{*}, g\left(t_{*}, \mu_{*}\right)\right] \subset W_{1}$, we have $\bar{w} \in\left[g\left(t_{*}, \mu_{*}\right), v\right]$, where $v$ is the point of intersection of the arc $[\alpha, \beta]$ and the line segment $\left[w_{*}, g\left(t_{*}, \mu_{*}\right)\right]$. Since $\left\|\bar{w}-g\left(t_{*}, \mu_{*}\right)\right\|=1$, then $\left\|v-g\left(t_{*}, \mu_{*}\right)\right\|>1$. We will now show that $\Delta t>0$ could have been assumed smaller from the beginning to eliminate the latter possibility. This will complete the proof of the invariance of $W$.

Reducing $\Delta \mathrm{t}$ : we claim that $\Delta t>0$ can be diminished further, so that

$$
\begin{aligned}
& \|v-g(t, \mu)\| \leq 1, \quad \text { for all } t \in\left[t_{\mu}, t_{\mu}+\tau\right], v \in[\alpha, \beta], \\
& \qquad \xi \notin B+g\left(t_{\mu}+\tau, \mu\right), \tau \in(0, \Delta t], \mu \in M .
\end{aligned}
$$

The square of $\|v-g(t, \mu)\|$ can be computed as

$$
r(t)=\langle v-g(t, \mu), N\rangle^{2}+\left\langle v-g(t, \mu), N^{\perp}\right\rangle^{2} .
$$

We have

$r^{\prime}(t)=-2\langle v-g(t, \mu), N\rangle \cdot\left\langle g_{t}^{\prime}(t, \mu), N\right\rangle-2\left\langle v-g(t, \mu), N^{\perp}\right\rangle \cdot\left\langle g_{t}^{\prime}(t, \mu), N^{\perp}\right\rangle$.

By diminishing $\Delta t>0$, we can make $\langle v-g(t, \mu), N\rangle$ as close to 1 as necessary $^{2}$. In addition, by the initial definition of $\Delta t$, we have $\left\langle g_{t}^{\prime}\left(t_{\mu}, \mu\right), N\right\rangle<0$ for all $t \in\left[t_{\mu}, t_{\mu}+\Delta t\right], \mu \in M$. Therefore, $\Delta t>0$ can be reduced to ensure

$$
\begin{aligned}
& \left.\min _{\substack{t \in\left[t_{\mu}, t_{\mu}+\tau\right], v \in[\alpha, \beta], \xi \notin B+g\left(t_{\mu}+\tau, \mu\right)}}\left[-\langle v-g(t, \mu), N\rangle \cdot\left\langle g_{t}^{\prime}(t, \mu), N\right\rangle\right]=c\right\rangle 0 . \\
& \xi \notin B+g\left(t_{\mu}+\tau, \mu\right), \\
& \tau \in(0, \Delta t], \mu \in M
\end{aligned}
$$

\footnotetext{
${ }^{2}$ Specifically, given $\gamma>0$ the value $\Delta t>0$ can be chosen so small that $|\langle v-g(t, \mu), N\rangle-1|<\gamma$ for all $t \in\left[t_{\mu}, t_{\mu}+\tau\right], v \in[\alpha, \beta], \xi \notin B+g\left(t_{\mu}+\tau, \mu\right), \tau \in(0, \Delta t]$, $\mu \in M$.
} 
On the other hand, by diminishing $\Delta t>0$ we can make $\left|\left\langle v-g(t, \mu), N^{\perp}\right\rangle\right|$ as small as necessary. Let us diminish $\Delta t>0$ to ensure

$$
\max _{\substack{t \in\left[t_{\mu}, t_{\mu}+\tau\right], v \in[\alpha, \beta], \xi \notin B+g\left(t_{\mu}+\tau, \mu\right), \tau \in(0, \Delta t], \mu \in M}}\left|-\left\langle v-g(t, \mu), N^{\perp}\right\rangle \cdot\left\langle g_{t}^{\prime}(t, \mu), N^{\perp}\right\rangle\right|<\frac{c}{2} .
$$

This choice of $\Delta t$ leads to

$r^{\prime}(t)>0, t \in\left[t_{\mu}, t_{\mu}+\tau\right], v \in[\alpha, \beta], \xi \notin B+g\left(t_{\mu}+\tau, \mu\right), \tau \in(0, \Delta t], \mu \in M$.

Since $r\left(t_{\mu}+\tau\right)=\left\|v-g\left(t_{\mu}+\tau\right)\right\|^{2}=1$ by the definition of arc $[\alpha, \beta]$, one can conclude that $r(t)<1$ for any $t \in\left[t_{\mu}, t_{\mu}+\tau\right)$ and (10) follows.

The proof of the fact that $\operatorname{proj}(W, B+g(t, \mu)) \subset W$ for any $t \in\left[t_{\mu}, t_{\mu}+\tau\right]$, $\xi \notin B+g\left(t_{\mu}+\tau, \mu\right), \tau \in(0, \Delta t], \mu \in M$ is now complete.

According to the theorem 2 from Kunze \& Monteiro Marques [17], the solution $t \mapsto v(t)$ of $(8)$ is the limit $\lim _{n \rightarrow \infty} v_{n}(t)$ of piecewise linear functions with vertexes given by

$$
\left\{\begin{aligned}
v_{i+1}^{n} & =\operatorname{proj}\left(v_{i}^{n}, B+g\left(\frac{i+1}{n} \tau+t_{\mu}\right)\right), \quad \text { for any } i \in \overline{0, n-1} \\
v_{0}^{n} & =\xi
\end{aligned}\right.
$$

Therefore, given an arbitrary $\tau \in(0, \Delta t]$ we have $v(t) \in W$ for all $t \in\left[t_{\mu}, t_{\mu}+\right.$ $\tau]$, i.e.

$$
\left|\left\langle v\left(t_{\mu}+\tau\right)-\xi, N^{\perp}\right\rangle\right| \leq a,
$$

where $a=\left|\left\langle\alpha, N^{\perp}\right\rangle\right|$, see Fig. $4 \mathrm{~b}$.

It remains to show that $a \leq\left\|g\left(t_{\mu}+\tau\right)\right\| \cdot b$, where $b=\left|\left\langle N^{\perp}, g\left(t_{\mu}+\tau\right)\right\rangle\right|$.

1) Using the triangle $\left(g\left(t_{\mu}+\tau\right), \xi, 0\right)$ we have $\|\alpha-\xi\|+1 \leq\left\|g\left(t_{\mu}+\tau\right)\right\|+\|\xi\| \leq$ $\left\|g\left(t_{\mu}+\tau\right)\right\|+1$. Therefore, $\|\alpha-\xi\| \leq\left\|g\left(t_{\mu}+\tau\right)\right\|$.

2) By similarity of right triangles

$$
\frac{a}{b}=\frac{\|\alpha-\xi\|}{\|\alpha-\xi\|+1} \leq \frac{\left\|g\left(t_{\mu}+\tau\right)\right\|}{1}
$$

The proof of the lemma is complete.

Remark 1 It is well known (see e.g. Kunze \& Monteiro-Marques [17]) that the solution $v(t)$ of (8) changes by a factor of $\Delta t$ when the time change is $\Delta t$. Lemma 1 states that a suitable projection of the solution changes by a factor of $(\Delta t)^{2}$. A similar property of sweeping processes is a key fact in the proof of the existence of solutions to (8), see e.g. [17, Theorem 2, formula (23)]. 
Proof of theorem 1. Let $r>0$ be small enough to have

$$
\begin{aligned}
& \left\langle f_{0}(N(-r)), N(-r)^{\perp}\right\rangle>0, \quad\left\langle f_{0}(N(r)), N(r)^{\perp}\right\rangle<0, \\
& \left\langle f_{0}(N(-r)), N(-r)\right\rangle \neq 0, \quad\left\langle f_{0}(N(r)), N(r)\right\rangle \neq 0 .
\end{aligned}
$$

Choose $\delta_{0}>0$ so small that

$$
\begin{aligned}
& \left\langle f_{0}((1-\delta) N(-r)), N(-r)^{\perp}\right\rangle>0, \quad\left\langle f_{0}((1-\delta) N(r)), N(r)^{\perp}\right\rangle<0, \\
& \left\langle f_{0}((1-\delta) N(-r)), N(-r)\right\rangle \neq 0, \quad\left\langle f_{0}((1-\delta) N(r)), N(r)\right\rangle \neq 0
\end{aligned}
$$

for all $\delta \in\left[0, \delta_{0}\right]$. Choose $\varepsilon_{0}>0$ small enough to ensure

$$
\begin{aligned}
& \left\langle f_{\varepsilon}(t,(1-\delta) N( \pm r)), N( \pm r)\right\rangle \neq 0, \\
& \left\langle f_{\varepsilon}(t,(1-\delta) N( \pm r)), N( \pm r)^{\perp}\right\rangle \neq 0,
\end{aligned}
$$

for all $\delta \in\left[0, \delta_{0}\right], t \in[0, T], \varepsilon \in\left[0, \varepsilon_{0}\right]$. We want to use Lemma 1 in order to find $\Delta t>0$ such that

$$
\left\langle u(t)-u\left(t_{0}\right), N(-r)^{\perp}\right\rangle \leq 0, \quad t \in\left[t_{0}, t_{0}+\Delta t\right],
$$

for any solution $u$ of (7) with the initial condition

$$
u_{0}=u\left(t_{0}\right) \in\left[\left(1-\delta_{0}\right) N(-r), N(-r)\right],
$$

and arbitrary $t_{0} \in[0, T]$ and $\varepsilon \in\left[0, \varepsilon_{0}\right]$. Observe (see e.g. Castaing \& Monteiro Marques [10, theorem 4.1]) that $u$ is the solution to

$$
\left\{\begin{aligned}
-\dot{v}(t) & \in N_{B+\int_{t_{0}}^{t} f_{\varepsilon}\left(s, \Omega\left(s, t_{0}, u_{0}\right)\right) d s}(v(t)), \\
v\left(t_{0}\right) & =u_{0}, \\
u(t) & =v(t)-\int_{t_{0}}^{t} f_{\varepsilon}\left(s, \Omega\left(s, t_{0}, u_{0}\right)\right) d s,
\end{aligned}\right.
$$

where $t \mapsto \Omega\left(t, t_{0}, u_{0}\right)$ is the solution of (7) that passes through $u_{0}$ at time $t_{0}$. To apply Lemma 1 we define $g: \mathbb{R} \times \mathbb{R}^{4} \rightarrow \mathbb{R}^{2}$ as

$$
g(t, \mu)=\int_{\mu_{1}}^{t} f_{\mu_{3}}\left(s, \Omega\left(s, \mu_{1}, \mu_{2}\right)\right) d s
$$

which takes the form $\int_{t_{0}}^{t} f_{\varepsilon}\left(s, \Omega\left(s, t_{0}, u_{0}\right)\right) d s$, when $\mu=\left(\begin{array}{c}t_{0} \\ u_{0} \\ \varepsilon\end{array}\right)$. Defining $\Delta \mathbf{t}$ : let $\Delta t>0$ be the one given by Lemma 1 applied with

$$
M=[0, T] \times\left[\left(1-\delta_{0}\right) N(-r), N(-r)\right] \times\left[0, \varepsilon_{0}\right]
$$


The conclusion of Lemma 1 is that

$$
\begin{aligned}
\left|\left\langle v(t)-v\left(t_{0}\right), N(-r)^{\perp}\right\rangle\right| \leq & \left\|\int_{t_{0}}^{t} f_{\varepsilon}\left(s, \Omega\left(s, t_{0}, u_{0}\right)\right) d s\right\| \\
& \cdot\left\langle\int_{t_{0}}^{t} f_{\varepsilon}\left(s, \Omega\left(s, t_{0}, u_{0}\right)\right) d s, N(-r)^{\perp}\right\rangle
\end{aligned}
$$

for any $t \in\left[t_{0}, t_{0}+\Delta t\right], v\left(t_{0}\right)=u_{0},\left(t_{0}, u_{0}, \varepsilon\right) \in M$. This yields

$$
\begin{aligned}
& \left\langle u(t)-u\left(t_{0}\right), N(-r)^{\perp}\right\rangle= \\
& =\left\langle v(t)-v\left(t_{0}\right)-\int_{t_{0}}^{t} f_{\varepsilon}\left(s, \Omega\left(s, t_{0}, u_{0}\right)\right) d s, N(-r)^{\perp}\right\rangle= \\
& =\left(\left\|\int_{t_{0}}^{t} f_{\varepsilon}\left(s, \Omega\left(s, t_{0}, u_{0}\right)\right) d s\right\|-1\right) \cdot\left\langle\int_{t_{0}}^{t} f_{\varepsilon}\left(s, \Omega\left(s, t_{0}, u_{0}\right)\right) d s, N(-r)^{\perp}\right\rangle .
\end{aligned}
$$

Diminish $\Delta t>0$ further to have

$$
\left\|\int_{t_{0}}^{t} f_{\varepsilon}\left(s, \Omega\left(s, t_{0}, u_{0}\right)\right) d s\right\| \leq \frac{1}{2}, \text { for all } t \in\left[t_{0}, t_{0}+\Delta t\right],\left(t_{0}, u_{0}, \varepsilon\right) \in M
$$

Taking into account (11) and (12) we finally get

$\left\langle u(t)-u\left(t_{0}\right), N(-r)^{\perp}\right\rangle \in$

$\in-\left\langle\int_{t_{0}}^{t} f_{\varepsilon}(s, u(s)) d s, N(-r)^{\perp}\right\rangle+$

$+\left[-\frac{1}{2}\left|\left\langle\int_{t_{0}}^{t} f_{\varepsilon}(s, u(s)) d s, N(-r)^{\perp}\right\rangle\right|, \frac{1}{2}\left|\left\langle\int_{t_{0}}^{t} f_{\varepsilon}(s, u(s)) d s, N(-r)^{\perp}\right\rangle\right|\right] \subset$

$\subset(-\infty, 0], \quad$ for all $t \in\left[t_{0}, t_{0}+\Delta t\right],\left(t_{0}, u_{0}, \varepsilon\right) \in M$.

Analogous arguments allow to conclude that

$$
\left\langle u(t)-u\left(t_{0}\right), N(r)^{\perp}\right\rangle \geq 0, \quad t \in\left[t_{0}, t_{0}+\Delta t\right],
$$

for any solution $u$ of $(7)$ with initial condition $u\left(t_{0}\right) \in\left[\left(1-\delta_{0}\right) N(r), N(r)\right]$ and any $t_{0} \in[0, T], \varepsilon \in\left[0, \varepsilon_{0}\right]$ provided that $\Delta t>0$ is sufficiently small.

Let us now consider a solution $u$ of (7) with the initial condition

$$
u_{0}=u\left(t_{0}\right) \in\left[\left(1-\delta_{0}\right) N(-r),\left(1-\delta_{0}\right) N(r)\right] .
$$

Diminish $\Delta t>0$ further to have

$$
\left\|\int_{t_{0}}^{t} f_{\varepsilon}\left(s, \Omega\left(s, t_{0}, u_{0}\right)\right) d s\right\|<\delta_{0}
$$


for any $t_{0} \in[0, T-\Delta t], t \in\left[t_{0}, t_{0}+\Delta t\right], u_{0} \in U, \varepsilon \in\left[0, \varepsilon_{0}\right]$. This ensures that $v(t)=u_{0}$ for all $t \in\left[t_{0}, t_{0}+\Delta t\right]$, where $v$ is the solution of (15) and $u_{0}$ is from (17). Therefore,

$$
\left\langle u(t)-u\left(t_{0}\right), N(0)\right\rangle=-\left\langle\int_{t_{0}}^{t} f_{\varepsilon}\left(s, \Omega\left(s, t_{0}, u_{0}\right)\right) d s, N(0)\right\rangle .
$$

Due to (6), we don't lose generality of the proof by considering $r>0, \delta_{0}>0$ and $\varepsilon_{0}>0$ so small that

$$
\left\langle f_{\varepsilon}\left(t, \Omega\left(t, t_{0}, u_{0}\right)\right), N(0)\right\rangle<0,
$$

for all $t_{0} \in[0, T-\Delta t], t \in\left[t_{0}, t_{0}+\Delta t\right], u_{0}$ from (17) and $\varepsilon \in\left[0, \varepsilon_{0}\right]$. Therefore, (18) implies that

$$
\left\langle u(t)-u\left(t_{0}\right), N(0)\right\rangle \geq 0
$$

for $u\left(t_{0}\right)$ satisfying (17).

The estimates (14), (16) and (19) ensure that none of the solutions of (7) with $u(0) \in U$ can leave $U$ during $[0, T]$. This completes the proof of the theorem.

The proof of Theorem 1 can be carried out through a direct application of the Moreau discretization scheme with no resort to papers by Kunze \& MonteiroMarques [17] and Castaing \& Monteiro-Marques [10]. Our goal was to restrict the paper to those aspects of justification that are essentially new (as much as required for a rigorous proof).

\section{Existence of periodic solutions}

A possible positive parameterization of the ball $B$ is given by $N_{0}(r)=\left(\begin{array}{l}-\sin r \\ \cos r\end{array}\right)$.

The function $\bar{f}$ is thus $\bar{f}(r)=\left\langle f_{0}\left(N_{0}(r)\right), N_{0}(r)^{\perp}\right\rangle$. Theorem 1 allows to draw the following corollary on the existence of $T$-periodic solutions to (7).

Corollary 1 Let $f_{\varepsilon}$ be $C^{1}$ for all $\varepsilon \in \mathbb{R}$ and assume that $f_{\varepsilon}(t, u) \rightarrow f_{0}(u)$ as $\varepsilon \rightarrow 0$ uniformly in $t \in[0, T]$. If for some $r_{0} \in \mathbb{R}$ one has

$$
f_{0}\left(N_{0}\left(r_{0}\right)\right)=-\lambda N_{0}\left(r_{0}\right) \text { with some } \lambda>0 \text { and } \bar{f}^{\prime}\left(r_{0}\right)<0 \text {, }
$$

then, for all $\varepsilon>0$ sufficiently small, the sweeping process (7) admits a Tperiodic solution $u_{\varepsilon}(t)$ such that

$$
u_{\varepsilon}(t) \rightarrow N_{0}\left(r_{0}\right) \quad \text { as } \quad \varepsilon \rightarrow 0 \quad \text { uniformly on }[0, T] .
$$


To prove the statement one has to consider a sequence $\delta_{k} \rightarrow 0$ as $k \rightarrow \infty$ along with the sequence of the respective values of $\varepsilon_{0}$ given by Theorem 1 which will now be denoted by $\varepsilon_{k}$. Given any $\varepsilon \in\left(0, \varepsilon_{k}\right]$, Theorem 1 ensures the existence of a $T$-periodic solution $u_{\delta_{k}, \varepsilon}([0, T]) \subset U$ to the sweeping process $(7)$ where $U$ has to be taken with $\delta=\delta_{k}$. Without loss of generality we can assume that $\varepsilon_{k} \rightarrow 0$ as $k \rightarrow \infty$ monotonically. The required solution $u_{\varepsilon}$ can now be defined for all $\varepsilon>0$ sufficiently small by

$$
u_{\varepsilon}(t)=u_{\delta_{k}, \varepsilon}(t), \quad \varepsilon \in\left[\varepsilon_{k}, \varepsilon_{k+1}\right), k \in \mathbb{N} .
$$

We recall that a function $g: \mathbb{R}^{2} \rightarrow \mathbb{R}^{2}$ is called real-analytic on an open set $W$, if for each $x_{*} \in \mathbb{R}^{2}$ there exists $r>0$ such that if $\left\|x-x_{*}\right\|<r$ then

$$
g_{j}(x)=\sum_{\alpha \in \mathbb{N}^{2}} g_{\alpha, j}\left(x_{1}-x_{*, 1}\right)^{\alpha_{1}} \cdot\left(x_{2}-x_{*, 2}\right)^{\alpha_{2}}, \quad j=1,2,
$$

where $g_{\alpha, j}$ are suitable numbers (see $[26, \S 1.1]$ ).

Corollary 2 Let $f_{\varepsilon}$ be $C^{1}$ for all $\varepsilon \in \mathbb{R}$ and assume that $f_{\varepsilon}(t, u) \rightarrow f_{0}(u)$ as $\varepsilon \rightarrow 0$ uniformly in $t \in[0, T]$. If $f_{0}$ is real-analytic in a neighborhood of the unit ball $B$ and for $r_{1}<r_{2}$ one has

$$
\left\langle f_{0}\left(N_{0}(r)\right), N_{0}(r)\right\rangle<0, r \in\left[r_{1}, r_{2}\right], \quad \bar{f}\left(r_{1}\right)>0, \quad \text { and } \bar{f}\left(r_{2}\right)<0,
$$

then there exists $r_{0} \in\left(r_{1}, r_{2}\right)$, such that for all $\varepsilon>0$ sufficiently small the sweeping process (7) admits a T-periodic solution $u_{\varepsilon}(t)$ that satisfies (21).

Real-analyticity is here used to conclude the existence of $r_{0} \in\left(r_{1}, r_{2}\right)$ at which $\bar{f}$ vanishes and strictly decreases.

\section{Conclusion}

In this paper we introduced a notion of an equilibrium of an autonomous sweeping process. We proved that, similar to what is happening in ordinary differential equations (see Cronin [11]), the stability of an equilibrium implies stability with respect to periodic perturbations. The goal of this paper was to identify the framework that can lead to a bifurcation theory for Moreau sweeping processes, not to make proofs in the most general possible settings (in contrast, we attempted to keep them as short as possible). The result can be extended to Lipschitz right-hand-sides in (6) and to time- and statedependent set $B$. At the same time, we don't yet understand how our result extends to BV sweeping processes.

Following the lines of Buica, Makarenkov \& Llibre [7], one can establish uniqueness and asymptotic stability of the periodic solution in theorem 1 . The ideas of Kamenskii, Makarenkov \& Nistri [14] and Buica, Llibre \& Makarenkov [7] are useful in the study of the response of a family of equilibria on $\partial B$ to periodic perturbations. However, most importantly, assuming $\bar{f}^{\prime}(0)=0$ leads to bifurcations, i.e. allows to design sweeping processes with different dynamic behavior. 
Acknowledgments

The first author was supported by RFBR Grants 14-01-00867 and 14-01-92004. The second author was supported by NSF Grant CMMI-1436856 and by RFBR Grant 13-01-00347. This work started when the second author worked as a Researcher at BCAM - Basque Center for Applied Mathematics (Bilbao, Spain) and when the first author benefited from a temporarily professorship at the same center. Both the authors express BCAM their deep gratitude for the excellent working conditions and warm hospitality. We finally acknowledge, that advises and critical remarks of anonymous referees were of great help for this paper.

\section{References}

1. J.-P. Aubin, A. Cellina, Differential inclusions. Set-valued maps and viability theory. Fundamental Principles of Mathematical Sciences, 264. Springer-Verlag, Berlin (1984)

2. D. Azzam-Laouir, S. Izza, L. Thibault, Mixed Semicontinuous Perturbation of Nonconvex State-Dependent Sweeping Process, Set-Valued Var. Anal 22, 271-283 (2014)

3. P. Ballard, The Dynamics of Discrete Mechanical Systems with Perfect Unilateral Constraints, Arch. Rational Mech. Anal. 154, 199-274 (2000).

4. I. Berstein, A. Halanai, Index of a singular point and the existence of periodic solutions of systems with small parameter, Dokl. Akad. Nauk SSSR (N.S.) 111, 923-925 (1956)

5. M. Bounkhel, L. Thibault, Nonconvex sweeping process and prox-regurality in Hilbert spaces. J. Nonlinear Convex Anal. 6, 359-374 (2005)

6. B. Brogliato, Nonsmooth Mechanics: Models, Dynamics and Control, Springer (1999)

7. A. Buica, J. Llibre, O. Makarenkov, Asymptotic stability of periodic solutions for nonsmooth differential equations with application to the nonsmooth van der Pol oscillator. SIAM J. Math. Anal. 40, 2478-2495 (2009)

8. A. Buica, J. Llibre, O. Makarenkov, Bifurcations from nondegenerate families of periodic solutions in Lipschitz systems. J. Differential Equations 252, 3899-3919 (2012)

9. A. Capietto, J. Mawhin, F. Zanolin, Continuation theorems for periodic perturbations of autonomous systems. Trans. Amer. Math. Soc. 329, 41-72 (1992)

10. C. Castaing, M. Monteiro Marques, BV periodic solutions of an evolution problem associated with continuous moving convex sets, Set-Valued Anal. 3, 381-399 (1995)

11. J. Cronin, Periodic solutions of some nonlinear differential equations. J. Differential Equations 3, 31-46 (1967)

12. P. Dupuis, H. Ishii, On lipschitz continuity of the solution mapping to the skorokhod problem, with applications, Stochastics and Stochastic Reports 35, 31-62 (1991)

13. M. Kamenskii, V. Obukhovskii, P. Zecca, Condensing multivalued maps and semilinear differential inclusions in Banach spaces. De Gruyter Series in Nonlinear Analysis and Applications, 7. Walter de Gruyter \& Co., Berlin (2001)

14. M. Kamenskii, O. Makarenkov, P. Nistri, A continuation principle for a class of periodically perturbed autonomous systems. Math. Nachr. 281, 42-61 (2008)

15. P. Krejci, A. Vladimirov, Polyhedral Sweeping Processes with Oblique Reflection in the Space of Regulated Functions, Set-Valued Analysis 11, 91-110 (2003)

16. P. Krejci, T. Roche, Lipschitz continuous data dependence of sweeping processes in BV spaces. Discrete Contin. Dyn. Syst. Ser. B 15, 637-650 (2011).

17. M. Kunze, M. Monteiro Marques, An introduction to Moreau's sweeping process. Impacts in mechanical systems (Grenoble, 1999), Lecture Notes in Phys., 551, Springer, Berlin, 1-60 (2000)

18. R. I. Leine, N. van de Wouw, Stability and convergence of mechanical systems with unilateral constraints, Lecture Notes in Applied and Computational Mechanics, 36. Springer-Verlag, Berlin (2008) 
19. M. D. P. Monteiro Marques, Differential inclusions in nonsmooth mechanical problems. Shocks and dry friction. Progress in Nonlinear Differential Equations and their Applications, 9. Birkhauser Verlag, Basel (1993)

20. J. J. Moreau, Evolution Problem Associated with a Moving Convex Set in a Hilbert Space, J. Differential Equations 26, 347-374 (1977)

21. J. J. Moreau, Application of convex analysis to the treatment of elastoplastic systems. In: Germain P., Nayroles B. (eds.) Applications of methods of functional analysis to problems in mechanics. Lecture notes in mathematics. 503. Springer, 56-89 (1976)

22. L. Paoli, A proximal-like method for a class of second order measure-differential inclusions describing vibro-impact problems, J. Differential Equations 250, 476-514 (2011)

23. L. Paoli, Time Discretization of Vibro-Impact, Phil. Trans. R. Soc. Lond. A. 359, $2405-$ $2428(2001)$

24. L. Paoli, Continuous dependence on data for vibro-impact problems. Math. Models Methods Appl. Sci. 15, 53-93 (2005)

25. V. Recupero, A continuity method for sweeping processes. J. Differential Equations 251, 2125-2142 (2011)

26. S. Krantz, H. R. Parks, A primer of real analytic functions. Second edition. Birkhauser Boston, Inc., Boston, MA, 2002. xiv+205 pp.

27. D. E. Stewart, Convergence of a time-stepping scheme for rigid-body dynamics and resolution of Painlev's problem. Arch. Ration. Mech. Anal. 145, 215-260 (1998)

28. D. E. Stewart, Rigid-Body Dynamics with Friction and Impact, SIAM Review 42, 3-39 (2000)

29. D. E. Stewart, Reformulations of Measure Differential Inclusions and Their Closed Graph Property, Journal of Differential Equations 175, 108-129 (2001)

30. L. Thibault, Sweeping process with regular and nonregular sets, J. Differential Equations 193, 1-26 (2003)

31. A. A. Tolstonogov, Sweeping process with unbounded nonconvex perturbation, Nonlinear Analysis 108, 291-301 (2014)

32. M. Valadier, Lipschitz approximation of the sweeping (or Moreau) process, J. Differential Equations 88, 248-264 (1990) 